\title{
Uso de vermicomposto favorece o crescimento de mudas de gravioleira (Annona muricata L. 'Morada') associadas a fungos micorrízicos arbusculares
}

\author{
Danielle Karla Alves da Silva1 ${ }^{1}$, Fábio Sérgio Barbosa da Silva ${ }^{1}$, Adriana Mayumi Yano-Melo² e Leonor Costa Maia ${ }^{1,3}$
}

Recebido em 3/04/2007. Aceito em 26/11/2007

\begin{abstract}
RESUMO - (Uso de vermicomposto favorece o crescimento de mudas de gravioleira (Annona muricata L. 'Morada') associadas a fungos micorrízicos arbusculares). A gravioleira, cujos frutos apresentam elevado potencial para exportação, é de fácil adaptação ao Semi-Árido nordestino, e a sua produção, economicamente importante, vem sendo estimulada. O uso de adubos orgânicos associados à inoculação com fungos micorrízicos arbusculares (FMA) pode ser útil na formação de mudas frutíferas, porém o efeito da adição de adubo na simbiose com gravioleira (Annona muricata L. 'Morada') não é conhecido. Foi investigado o efeito de FMA multiplicado em substrato com resíduo orgânico na formação de mudas de gravioleira mantidas em substratos com fertilizante. O delineamento foi inteiramente casualizado, em fatorial de 2×5: 2 substratos (solo sem ou com 10\% de vermicomposto) e cinco tratamentos de inoculação (Acaulospora longula Spain \& Schenck e Gigaspora albida Schenck \& Smith produzidos em substratos com ou sem resíduo orgânico e controle não inoculado), com quatro repetições. Após 102 dias, avaliou-se: massa seca da parte aérea e radicular, altura, diâmetro do caule, taxa de crescimento, produção de esporos de FMA e de glomalina, atividade enzimática do solo, respiração microbiana, colonização micorrízica total, arbuscular e hifálica. Em geral, a inoculação com FMA estimulou o crescimento, mas no tratamento adubado as mudas em simbiose com G. albida não foram beneficiadas pela associação. O uso de vermicomposto estimulou a colonização micorrízica, a respiração microbiana, a atividade enzimática e a produção de glomalina no solo, porém reduziu a esporulação de A. longula. A utilização de FMA e vermicomposto pode constituir alternativa na produção de mudas de gravioleira, pois reduziu à metade o tempo de formação das mudas e pode reduzir em $75 \%$ a dose de adubo a ser aplicada. No entanto, a escolha de FMA compatíveis com o hospedeiro é indispensável para garantir respostas positivas. A aplicação conjunta de FMA e adubo orgânico também pode melhorar a qualidade do solo, contribuindo para a produção sustentável de mudas desta e de outras fruteiras.
\end{abstract}

Palavras-chave: adubação orgânica, Glomeromycota, micorriza, produção de mudas

\begin{abstract}
Use of earthworm manure improves growth of soursop seedlings (Annona muricata L. 'Morada') associated with arbuscular mycorrhizal fungi). Annona muricata L. (soursop) easily adapts to irrigation in the semiarid Northeast. The economically important fruits have high exportation potential, so production has been encouraged. The use of organic amendments, together with arbuscular mycorrhizal fungi (AMF) may be useful for production of seedlings. However, the effect of such amendments on the symbiosis established between AMF and soursop remains unknown. The effect of AMF multiplied in a substrate with earthworm manure on the formation of A. muricata seedlings maintained in fertilized substrate was investigated. The experimental design was entirely random, in a factorial of $2 \times 5=2$ substrates (with or without $10 \%$ organic manure) and five inoculation treatments (Acaulospora longula Spain \& Schenck and Gigaspora albida Schenck \& Smith, produced in substrates with or without organic residue and an uninoculated control), with four replicates. After 102 days, dry mass of shoots and roots, height, growth rate, production of AMF spores and glomalin, soil enzymatic activity, microbial respiration, total, arbuscular and hyphal colonization were evaluated. In general, seedling growth was stimulated by the inoculation with AMF, but in the fertilized soil, growth of seedlings in symbiosis with G. albida was not benefited. The use of organic fertilizer stimulated mycorrhizal colonization, microbial respiration, enzymatic activity and glomalin production, but inhibited $A$. longula sporulation. The application of AMF and organic manure may constitute an alternative for production of Annona muricata, since it reduced by half the period for seedling formation, and can reduce by $75 \%$ the dose of fertilizer to be applied. However, the choice of AMF compatible with the host is needed to assure positive results. The combination of both, AMF and organic amendment, can also improve soil quality, thus contributing to sustainable production of seedlings of A. muricata and other fruit trees.
\end{abstract}

Key words: organic amendment, Glomeromycota, mycorrhiza, seedling production

\section{Introdução}

Os fungos micorrízicos arbusculares (FMA) constituem associação com a maioria das plantas podendo atuar como ferramenta biotecnológica importante na agricultura, pois a simbiose aumenta a área de absorção de nutrientes pela planta, conferindo ao vegetal maior crescimento (Costa et al. 2005) e tolerância a estresses de origem biótica e abiótica (Azcón-Aguilar \& Barea 1997). Além dos benefícios

\footnotetext{
1 Universidade Federal de Pernambuco, Departamento de Micologia, Centro de Ciências Biológicas, Rua Prof. Nelson Chaves s.n., Cidade Universitária, 50670-420 Recife, PE, Brasil

2 Universidade Federal do Vale do São Francisco, Departamento de Zootecnia, Rodovia BR 407, Km 8, Jardim São Paulo, 56314-520 Petrolina, PE, Brasil

3 Autor para correspondência: leonorcmaia@yahoo.com.br
} 
nutricionais para o hospedeiro, os FMA produzem no micélio externo uma glicoproteína (glomalina) que favorece a estabilidade de agregados no solo (Wright \& Upadhyaya 1998), contribuindo para melhoria da qualidade edáfica.

A necessidade de sistemas agrícolas sustentáveis tem impulsionado a busca por práticas agrícolas que, além de favorecer a produtividade de culturas, não comprometam a qualidade do solo; uma das alternativas é o uso de adubos orgânicos, que aumentam a fertilidade e a estruturação do solo (Caravaca et al. 2002) e são amplamente empregados no substrato para formação de mudas. Quando aplicados em doses adequadas esses fertilizantes favorecem a microbiota do solo, benefício que pode ser evidenciado pela maior atividade respiratória e enzimática em solos adubados (Marschner et al. 2003; Roldán et al. 2003; García-Gil et al. 2004). Maiores benefícios no uso de adubos podem ser alcançados pelo emprego de FMA, sendo conhecido o efeito sinérgico da aplicação de resíduos orgânicos e da inoculação micorrízica no crescimento vegetal (Caravaca et al. 2003). Por outro lado, a efetividade de FMA multiplicados em substratos orgânicos deve ser investigada, pois estes também podem constituir alternativa de baixo custo para produção de inóculo micorrízico.

A gravioleira é a mais tropical das Annonaceae, sendo de fácil adaptação ao Semi-Árido nordestino, em cultivo irrigado, o que possibilita sua produção e comercialização, principalmente pelo potencial econômico (Ramos et al. 2001). Como o tempo de produção de mudas de gravioleira é longo (oito meses), a utilização de FMA tem sido apontada como alternativa para redução do tempo de transplantio ao campo (Chu et al. 2001). Porém, em substrato com resíduos orgânicos o efeito da micorrização de gravioleiras não é conhecido. A utilização de FMA adaptados a diversas condições edáficas em geral produz melhores respostas de crescimento vegetal (Calvente et al. 2004; Caravaca et al. 2005).

Os objetivos deste trabalho foram verificar o efeito de FMA, produzidos em substratos com resíduo orgânico, na formação de mudas de gravioleira e avaliar o impacto do uso combinado da inoculação micorrízica e da adubação orgânica na atividade microbiana do solo.

\section{Material e métodos}

Substratos - Para a produção dos inóculos: solo do tipo Franco-argilo-arenoso, proveniente de Camaragibe, PE. Para o experimento: Solo do tipo arenoso, proveniente da Estação Experimental da Empresa Pernambucana de Pesquisa Agropecuária (IPA) em Itapirema (Goiana, PE), foi peneirado, desinfestado com Bromex ${ }^{\circledR}(98 \%$ Brometo de Metila e 2\% de cloropicrina) por 5 dias e utilizado após 15 dias, quando se adicionou $10 \%$ de vermicomposto (v:v) (Tab. 1), adubo de baixo custo recomendado para produção de mudas de gravioleira (Souza et al. 2003).

FMA - Inóculo de Gigaspora albida Schenck \& Smith (UFPE 01) foi produzido em: (a) solo; (b) solo com $10 \%$ de composto orgânico; inóculo de Acaulospora longula Spain \& Schenck (UFPE 21) foi produzido em: (a) solo; (b) areia com 10\% de terra vegetal previamente tratados para produção de inóculos (Tab. 1). Esses substratos foram os mais promissores em seleção prévia para produção de inóculo dos FMA testados. Como hospedeiro para obtenção de inóculo dos dois fungos foi utilizado painço (Panicum miliaceum L.).

Material vegetal - Sementes de fruto maduro de Annona muricata L. 'Morada' foram desinfestadas com hipoclorito de sódio $(0,05 \%)$ por 15 minutos, lavadas, embebidas por 24 horas em água destilada esterilizada e colocadas para germinar em copos de $180 \mathrm{~mL}$ (2 sementes por copo) com vermiculita esterilizada $\left(121^{\circ} \mathrm{C} / 30 \mathrm{~min}\right)$. As plantas obtidas foram utilizadas nos experimentos.

Inoculação - Solo-inóculo dos FMA (correspondendo a 200 esporos/pote) foi depositado na região das raízes de plântulas de gravioleira com duas folhas definitivas, em copos de $300 \mathrm{~mL}$ contendo $260 \mathrm{~mL}$ de substrato. As plantas permaneceram por 15 dias nos copos, sendo posteriormente transferidas para sacos pretos de polietileno $(14 \times 27 \mathrm{~cm}) \mathrm{com} 1,8 \mathrm{~kg}$ de solo.

Delineamento experimental - Foi do tipo inteiramente casualizado em arranjo fatorial de $2 \times 5: 2$ tratamentos de adubação (com ou sem vermicomposto) $\times 5$ tratamentos de inoculação (inóculos de Acaulospora longula e Gigaspora albida, produzidos em solo ou em substratos com resíduo orgânico e tratamento controle, sem inoculação), com 4 repetições, totalizando 40 unidades experimentais.

Condições experimentais - $\mathrm{O}$ experimento foi mantido em telado sob condições ambientais de umidade $\left(\mathrm{UR}_{\min }\right.$ $30,9 \%$; $\left.\mathrm{UR}_{\max } 71,5 \%\right)$, temperatura $\left(\mathrm{T}_{\min } 28{ }^{\circ} \mathrm{C} ; \mathrm{T}_{\text {máx }}\right.$ $36,3{ }^{\circ} \mathrm{C}$ ) e luminosidade. A irrigação foi feita diariamente de modo a manter $50 \%$ do volume dos poros (VTP) preenchidos com água.

Avaliações - Após 102 dias da inoculação foram avaliados: altura, diâmetro do caule, massa seca da parte aérea (MSPA), massa seca da parte subterrânea (MSPS), taxa de crescimento relativa (TCR mensal), densidade de esporos, colonização micorrízica total, hifálica e arbuscular, atividade enzimática geral, respiração microbiana e produção de glomalina. A matéria seca foi 
Tabela 1. Caracterização química dos substratos utilizados para multiplicação dos fungos micorrízicos arbusculares (FMA) e para cultivo da gravioleira (Annona muricata L. 'Morada'). CTC = capacidade de troca cationnica; CO = composto orgânico; TV= terra vegetal; $\mathrm{VC}=$ vermicomposto

\begin{tabular}{|c|c|c|c|c|c|c|}
\hline Substratos & $\begin{array}{c}\mathrm{pH} \\
\left(\mathrm{H}_{2} \mathrm{O}-1: 2,5\right)\end{array}$ & $\begin{array}{c}\mathrm{P} \\
\left(\mathrm{mg} \mathrm{dm}^{-3}\right)\end{array}$ & $\begin{array}{c}\mathrm{C} \\
\left(\mathrm{g} \mathrm{Kg}^{-1}\right)\end{array}$ & $\begin{array}{c}\mathrm{N} \\
\left(\mathrm{cmol}_{\mathrm{c}} \mathrm{dm}^{-3}\right)\end{array}$ & CTC & $\mathrm{C} / \mathrm{N}$ \\
\hline \multicolumn{7}{|c|}{ Produção de inóculo de FMA } \\
\hline Solo & 4,6 & 4 & 13,30 & 1,2 & 1,69 & 11,08 \\
\hline Solo $+10 \% \mathrm{CO}$ & 5,6 & 53 & 26,10 & 2,0 & 9,89 & 13,05 \\
\hline Areia $+10 \% \mathrm{TV}$ & 6,1 & 65 & 4,00 & 0,3 & 3,38 & 13,33 \\
\hline \multicolumn{7}{|c|}{ Cultivo de gravioleira } \\
\hline Solo & 5,4 & 5 & 20,99 & 1,9 & 8,40 & 11,05 \\
\hline Solo $+10 \% \mathrm{VC}$ & 5,5 & 70 & 20,65 & 1,9 & 9,53 & 10,86 \\
\hline
\end{tabular}

determinada após secagem do material em estufa de circulação de ar $\left(60^{\circ} \mathrm{C}\right)$ até peso constante; a TCR/mês foi calculada pela equação $\left(\mathrm{pr}^{2} \mathrm{x}\right.$ altura)/idade da planta, onde $r=1 / 2$ diâmetro do caule (Sieverding 1991). Os esporos foram quantificados em estereomicroscópio (40X), após extraídos dos substratos pela técnica de decantação e peneiramento úmido (Gerdemann \& Nicolson 1963; Jenkins 1964). A colonização micorrízica foi determinada (McGonigle et al. 1990) após diafanização das raízes e coloração com clorazol black E (Brundrett et al. 1984). A atividade enzimática geral do solo foi estimada pela hidrólise do diacetato de fluoresceína (FDA) (Swisher \& Carroll 1980), enquanto a respiração microbiana (emissão de $\mathrm{CO}_{2}$ ) foi medida por titulometria (Grisi 1978). A glomalina facilmente extraível (GFE) foi obtida (autoclave $121^{\circ} \mathrm{C} / 30 \mathrm{~min}$ ) em solução de citrato de sódio $(20 \mathrm{mM}$; pH 7,0) (Wright \& Upadhyaya 1998) a partir de agregados de solo (<1 mm diâm.) e dosada pelo método de Bradford (1976).

Análise estatística - Os valores de produção de esporos foram transformados em $\sqrt{x}+1$ e os de colonização (hifálica, arbuscular e total) em arco seno $\sqrt{ } \mathrm{x} / 100$. Os dados foram submetidos à análise de variância e as médias comparadas pelo teste de Tukey (5\%) utilizando o programa Statistica 5.0 (Statsoft 1997).

\section{Resultados}

Em geral, a inoculação com FMA estimulou o crescimento das mudas de gravioleira, especialmente em solo com $10 \%$ de vermicomposto. Nesta condição, a simbiose com A. longula multiplicado em solo promoveu a formação de mudas com maior altura, diâmetro do caule, massa seca da parte aérea e subterrânea e taxa de crescimento relativa. Em solo não fertilizado, com exceção da massa seca da parte aérea, maiores benefícios da micorrização foram evidenciados nas mudas em simbiose com o isolado de A. longula multiplicado nos dois substratos. Por outro lado, a inoculação com G. albida não favoreceu o crescimento das mudas e na maioria dos parâmetros avaliados os valores obtidos foram similares aos do controle sem fungo (Tab. 2).

A aplicação de vermicomposto estimulou a formação de arbúsculos no córtex radicular das mudas $(\mathrm{P}<0,05)$ que atingiu $2,5 \%$, em contraste com o tratamento não adubado $(0,8 \%)$. No entanto, apenas nas raízes associadas a G. albida houve estímulo da adubação na

Tabela 2. Crescimento de mudas de gravioleira (Annona muricata L. 'Morada') em solo sem ou com $10 \%$ de vermicomposto, 102 dias após a inoculação com fungos micorrízicos arbusculares. MSPA = massa seca da parte aérea; MSPS = massa seca da parte subterrânea; $\mathrm{TCR}=$ taxa de crescimento relativo $/ \mathrm{mês}$; $\mathrm{Al}-\mathrm{S}=$ Acaulospora longula produzido em solo $; \mathrm{Al}-\mathrm{Org}=$ A longula produzido em areia com $10 \%$ de terra vegetal; $\mathrm{Ga}-\mathrm{S}=$ Gigaspora albida produzido em solo; $\mathrm{Ga}-\mathrm{Org}=$ G. albida produzido em solo com $10 \%$ de composto orgânico; $\mathrm{VC}=$ vermicomposto.

\begin{tabular}{|c|c|c|c|c|c|}
\hline \multirow[t]{2}{*}{ Tratamentos } & \multicolumn{5}{|c|}{ Parâmetros } \\
\hline & $\begin{array}{l}\text { Altura } \\
(\mathrm{cm})\end{array}$ & $\begin{array}{l}\text { Diâmetro } \\
\text { do caule } \\
(\mathrm{cm})\end{array}$ & $\begin{array}{l}\text { MSPA } \\
(\mathrm{g})\end{array}$ & $\begin{array}{l}\text { MSPS } \\
(\mathrm{g})\end{array}$ & $\begin{array}{c}\text { TCR } \\
\text { (cm/mês) }\end{array}$ \\
\hline \multicolumn{6}{|l|}{ Solo } \\
\hline Controle & $19,92 \mathrm{c}$ & $0,31 \mathrm{~cd}$ & $0,86 \mathrm{e}$ & $0,40 \mathrm{c}$ & $0,46 \mathrm{~d}$ \\
\hline Al-S & $44,77 \mathrm{ab}$ & $0,57 \mathrm{~b}$ & $5,84 \mathrm{bc}$ & $2,24 \mathrm{ab}$ & $3,34 \mathrm{bc}$ \\
\hline Al-Org & $38,72 b$ & $0,56 b$ & $4,12 \mathrm{~d}$ & $1,84 \mathrm{~b}$ & $2,85 \mathrm{c}$ \\
\hline $\mathrm{Ga}-\mathrm{S}$ & $18,45 \mathrm{c}$ & $0,28 \mathrm{~d}$ & $0,81 \mathrm{e}$ & $0,32 \mathrm{c}$ & $0,35 \mathrm{~d}$ \\
\hline Ga-Org & $20,23 \mathrm{c}$ & $0,40 \mathrm{c}$ & $1,30 \mathrm{e}$ & $0,70 \mathrm{c}$ & $0,79 \mathrm{~d}$ \\
\hline \multicolumn{6}{|c|}{ Solo $+10 \%$ VC } \\
\hline Controle & $44,66 \mathrm{ab}$ & $0,65 b$ & $6,23 \mathrm{bc}$ & $1,85 b$ & $4,36 \mathrm{~b}$ \\
\hline $\mathrm{Al}-\mathrm{S}$ & $50,75 \mathrm{a}$ & $0,79 a$ & $7,83 \mathrm{a}$ & $2,90 \mathrm{a}$ & $7,45 \mathrm{a}$ \\
\hline Al-Org & $43,73 \mathrm{ab}$ & $0,62 b$ & $6,72 b$ & $2,28 \mathrm{ab}$ & $3,89 \mathrm{bc}$ \\
\hline $\mathrm{Ga}-\mathrm{S}$ & $39,70 \mathrm{~b}$ & $0,63 b$ & $5,38 \mathrm{c}$ & $1,99 \mathrm{~b}$ & $3,78 \mathrm{bc}$ \\
\hline Ga-Org & $35,26 \mathrm{~b}$ & $0,55 b$ & $4,05 \mathrm{~d}$ & $1,41 b c$ & $2,47 \mathrm{c}$ \\
\hline
\end{tabular}

Médias seguidas da mesma letra, na coluna, não diferem estatisticamente pelo teste de Tukey $(\mathrm{P}<0,05)$. 
colonização por hifas e total. A produção de esporos de G. albida foi extremamente baixa ou nula nos dois substratos (com e sem adubo), enquanto a de A. longula foi mais favorecida em solo sem adubo, sendo a reprodução drasticamente reduzida no tratamento com vermicomposto (Tab. 3).

Em relação aos parâmetros microbianos (emissão de $\mathrm{CO}_{2}$, atividade enzimática geral e glomalina), houve interação significativa entre FMA e adubação $(\mathrm{P}<0,01)$ apenas em relação à hidrólise do FDA, maior no substrato adubado (Tab. 3). Para os demais parâmetros não houve efeito da interação, embora maiores estimativas tenham sido registradas em solo com vermicomposto $(\mathrm{P}<0,01)$ (Fig. 1).

\section{Discussão}

O efeito sinérgico positivo da aplicação conjunta de adubo e de FMA, tal como observado, também foi referido para outros vegetais (Caravaca et al. 2004). Isso ocorre porque os substratos orgânicos usados para a formação de mudas, assim como os FMA, podem melhorar as características físico-químicas do solo (Caravaca et al. 2002).

Apesar de não existir especificidade de hospedeiro pelo FMA, existem diferentes graus de compatibilidade funcional entre os simbiontes (Costa et al. 2001), o que reflete no desenvolvimento dos parceiros. Isso foi observado neste trabalho, considerando que benefícios da micorrização ocorreram apenas quando as mudas estavam associadas a $A$. longula.

Em solos com baixa disponibilidade de nutrientes geralmente há incremento no crescimento do hospedeiro decorrente da micorrização (Chu et al. 2001; Anjos et al. 2005; Costa et al. 2005). Apesar da aplicação conjunta de vermicomposto e A. longula ter proporcionado a formação de mudas sadias, os maiores incrementos no crescimento da gravioleira foram obtidos nos tratamentos sem adição de resíduo orgânico, indicando que em solos com baixa disponibilidade de nutrientes ocorre máxima resposta à micorrização.

A formação de arbúsculos no córtex radicular das mudas foi estimulada no substrato adubado em todos os tratamentos de inoculação; no entanto apenas na simbiose com G.albida a colonização total e por hifas foi estimulada pela aplicação de vermicomposto. Gryndler et al. (2002) referiram aumento da colonização total produzida por espécies de Glomus em Plantago lanceolata L., em substrato enriquecido com celulose. Assim, a adubação pode favorecer tanto o hospedeiro quanto o fungo, contribuindo para o estabelecimento da simbiose. No entanto, o efeito positivo da adubação não se repetiu na colonização das raízes por A. longula, indicando diferenças na resposta dos FMA, tal como observado na associação entre Olea europaea L. e Rhamnus lycioides L. com Glomus intraradices Schenck \& Smith, em solo com composto orgânico (Caravaca et al. 2002).

Embora não tenha sido observada diferença na taxa de colonização total e hifálica por A. longula, o uso de inóculo deste fungo pode ser alternativa na formação de mudas de gravioleiras, pois, além de incrementar o crescimento, apresenta elevada taxa de reprodução no solo. Esta característica tem sido apontada (Abbott et al. 1994) como importante na seleção de inoculantes para aplicação na agricultura. Porém, no solo adubado com

Tabela 3. Colonização micorrízica, produção de esporos e atividade enzimática geral em solo não adubado ou adubado com 10\% de vermicomposto e cultivado com gravioleira (Annona muricata L. 'Morada') 102 dias após a inoculação com fungos micorrízicos arbusculares. Inóculos: Al-S = Acaulospora longula produzido em solo; Al-Org = A. longula produzido em areia com $10 \%$ de terra vegetal; Ga-S= Gigaspora albida produzido em solo; Ga-Org = G. albida produzido em solo com $10 \%$ de composto orgânico; VC = vermicomposto.

\begin{tabular}{|c|c|c|c|c|}
\hline Tratamentos & $\begin{array}{c}\text { Colonização total } \\
(\%)\end{array}$ & $\begin{array}{c}\text { Colonização hifálica } \\
(\%)\end{array}$ & $\begin{array}{l}\text { Densidade de esporos } \\
\text { (esporos } 100 \mathrm{~g}^{-1} \text { solo) }\end{array}$ & $\begin{array}{l}\text { Atividade enzimática geral } \\
\text { ( } \mu \text { g de FDA hidrolisado } \mathrm{g} \\
\text { solo } \operatorname{seco}^{-1} \mathrm{~h}^{-1} \text { ) }\end{array}$ \\
\hline \multicolumn{5}{|l|}{ Solo } \\
\hline S/FMA & - & - & - & $23,78 \mathrm{ab}$ \\
\hline Al-S & $13,51 \mathrm{a}$ & $10,89 \mathrm{ab}$ & $5885,00 \mathrm{a}$ & $28,63 \mathrm{ab}$ \\
\hline Al-Org & $15,80 \mathrm{a}$ & $15,39 \mathrm{a}$ & $4360,00 \mathrm{a}$ & $21,52 b$ \\
\hline Ga-S & $1,39 \mathrm{bc}$ & $1,15 \mathrm{c}$ & $15,00 \mathrm{~d}$ & $16,19 b$ \\
\hline Ga-Org & $0,20 \mathrm{c}$ & $0,20 \mathrm{c}$ & $7,00 \mathrm{~d}$ & $13,92 b$ \\
\hline \multicolumn{5}{|l|}{ Solo $+10 \%$ VC } \\
\hline S/FMA & - & - & - & $27,23 \mathrm{ab}$ \\
\hline Al-S & $7,73 \mathrm{a}$ & $6,24 \mathrm{ab}$ & $1846,00 \mathrm{~b}$ & $27,14 \mathrm{ab}$ \\
\hline Al-Org & $14,64 \mathrm{a}$ & $9,50 \mathrm{ab}$ & $568,00 \mathrm{c}$ & $30,89 a$ \\
\hline $\mathrm{Ga}-\mathrm{S}$ & $8,10 \mathrm{a}$ & $5,96 \mathrm{~b}$ & $1,00 \mathrm{~d}$ & $23,82 \mathrm{ab}$ \\
\hline Ga-Org & $5,83 \mathrm{ab}$ & $4,59 \mathrm{bc}$ & $0,00 \mathrm{~d}$ & $22,95 \mathrm{ab}$ \\
\hline
\end{tabular}

Médias seguidas da mesma letra, na coluna, não diferem estatisticamente pelo teste de Tukey $(\mathrm{P}<0,05)$. 

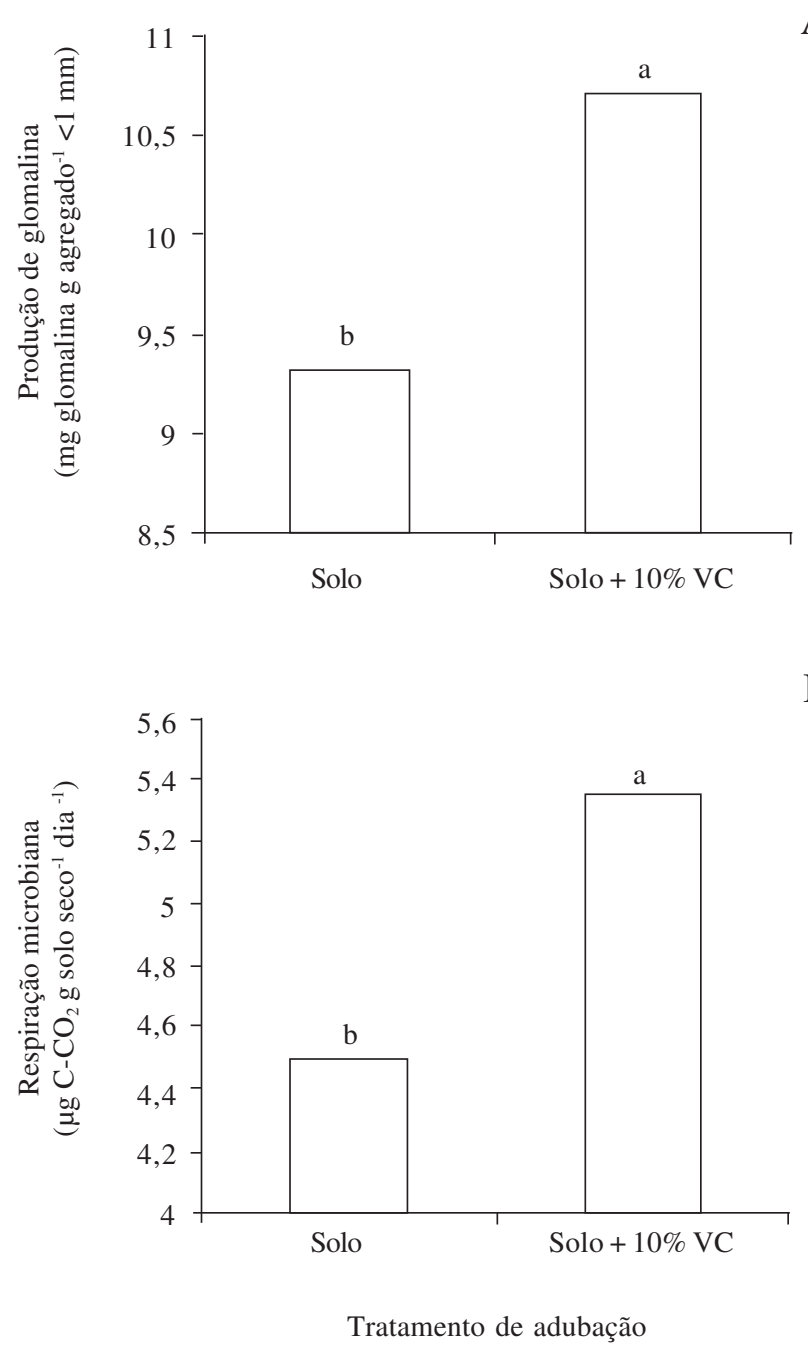

Figura 1. Produção de glomalina (A) e respiração microbiana (B) em solo não adubado ou adubado com $10 \%$ de vermicomposto (VC) utilizado para cultivo de gravioleira, independentemente do tratamento de inoculação, 102 dias após o início do experimento. Médias seguidas da mesma letra não diferem estatisticamente pelo teste de Tukey $(\mathrm{P}<0,05)$.

vermicomposto a esporulação de $A$. longula foi reduzida, o que possivelmente ocorreu devido ao elevado teor de $\mathrm{P}\left(70 \mathrm{mg} \mathrm{dm}^{-3}\right)$ neste substrato, fator que em geral inibe a produção de esporos (Gaur \& Adholeya 2000). Desse modo, é importante determinar a melhor dose de adubo a ser utilizada, para não inibir a esporulação dos FMA benéficos ao hospedeiro.

$\mathrm{O}$ fato da atividade microbiana (emissão de $\mathrm{CO}_{2} \mathrm{e}$ atividade enzimática geral) ter sido incrementada no solo adubado indica que o vermicomposto serviu como fonte de energia para os processos oxidativos microbianos, como sugerido por Medina et al. (2004). Este efeito é comumente referido quando doses e tipos adequados de resíduos são utilizados (Marschner et al. 2003), considerando que a matéria orgânica pode servir como fonte de energia nos processos microbianos (Carrasco et al. 2006). Além disso, a elevada atividade no substrato adubado pode ser decorrente do aumento da população de microrganismos neste resíduo e da atividade de enzimas aderidas aos colóides do vermicomposto empregado como adubo (Pascual et al. 1998).

A atividade microbiana não diferiu entre os tratamentos de inoculação até a colheita do experimento (102 dias), quando as mudas ainda não haviam atingido a fase de reprodução. Wamberg et al. (2003) registraram influência da micorrização de plantas de ervilha (Pisum sativum L.) na respiração microbiana apenas quando estas estavam em estádio reprodutivo. Supõe-se que nas fases mais avançadas da simbiose ocorre maior contribuição da rede hifálica extra-radicular neste processo (Caravaca et al. 2004).

A produção de hifas externas é favorecida quando fontes orgânicas são aplicadas ao solo (Palenzuela et al. 2002) e a maior produção de glomalina no substrato com adubo pode ter decorrido da elevada quantidade de hifas neste meio. Adicionalmente, o aumento da fertilidade do solo pode ter favorecido a produção deste composto (Lovelock et al. 2004), considerando que Wuest et al. (2005) registraram aumento na produção de glomalina em solo adubado com esterco bovino. Tal produção diferenciada no solo com adubo reflete melhoria na qualidade do solo, dada a estreita relação da glomalina com a agregação de partículas (Rillig 2004).

Em alguns casos, FMA adaptados ao ambiente onde serão aplicados têm melhor atuação sobre o hospedeiro (Calvente et al. 2004). Todavia, isso não ocorreu com A. longula: em solo adubado, o fungo multiplicado em substrato sem adubo produziu mais benefícios no hospedeiro do que o oriundo de substrato adubado. Resultados semelhantes foram registrados por Tian et al. (2004): um isolado de Glomus mosseae (Nicolson \& Gerd.) Gerd. \& Trappe de áreas salinizadas foi menos efetivo em condições de estresse salino que o fungo introduzido. Fidebilus et al. (2001) também observaram que a origem de isolados de Glomus não interferiu na eficiência do uso da água por Citrus volkameriana Tan. $\&$ Pasq.

Souza et al. (2003) referiram que mudas de gravioleira alcançaram altura para transplantio ao campo $(40 \mathrm{~cm}$ ) após 210 dias de cultivo em substrato constituído por $40 \%$ de vermicomposto, recebendo $5 \mathrm{Kg} \mathrm{P}_{2} \mathrm{O}_{5} \mathrm{~m}^{-3}$. No presente trabalho, mudas vigorosas para transplantio foram obtidas em apenas 102 dias da inoculação com A. longula em solo com $10 \%$ de vermicomposto, o que traduz redução de $75 \%$ no uso de adubo orgânico e metade do tempo para formação das mudas. Além disso, não foi necessária a aplicação de fertilizantes químicos, como sugerido para essa cultura (Barbosa et al. 2003), o que mostra a viabilidade 
de práticas agrícolas mais sustentáveis para produção de fruteiras.

A aplicação conjunta de FMA e adubos compostados tem efeito sinérgico positivo sobre o crescimento de gravioleiras, na dependência do isolado de FMA e da dosagem de adubo aplicado. Portanto, pode constituir alternativa na produção desta frutífera, com redução no tempo de formação de mudas e na dosagem de adubo. Além disso, a adoção desse sistema pode favorecer a qualidade do solo, contribuindo para produção sustentável de mudas de gravioleira.

\section{Agradecimentos}

Ao Conselho Nacional de Desenvolvimento Científico e Tecnológico (CNPq), pela concessão de bolsas PIBIC a Daniela Karla Alves da Silva e PQ a Leonor Costa Maia; à Coordenação de Aperfeiçoamento de Pessoal de Nível Superior (CAPES), pela concessão de bolsa de Doutorado (PG em Biologia de Fungos) a Fábio Sérgio Barbosa da Silva.

\section{Referências bibliográficas}

Abbott, L.K.; Robson, A.D. \& Gazey, C. 1994. Selection of inoculant vesicular-arbuscular mycorrhizal fungi. Pp. 461-481. In: J.R. Norris; D. Read \& A.K. Varma (eds.). Techniques for Mycorrhizal Research. London, Academic Press.

Anjos, E.C.T.; Cavalcante, U.M.T.; Santos, V.F. \& Maia. L.C. 2005. Produção de mudas de maracujazeiro-doce micorrizadas, em solo desinfestado e adubado com fósforo. Pesquisa Agropecuária Brasileira 40: 345-351.

Azcón-Aguilar, C. \& Barea, J.M. 1997. Applying mycorrhiza biotechnology to horticulture: significance and potentials. Scientia Horticulturae 68: 1-24.

Barbosa, Z.; Soares, I. \& Crisóstomo, L.A. 2003. Crescimento e absorção de nutrientes por mudas de gravioleira. Revista Brasileira de Fruticultura 25: 519-522.

Bradford, M.M. 1976. A rapid and sensitive method for the quantification of microgram quantities of protein utilizing the principle of protein-dye binding. Analytical Biochemistry 72: 248-254.

Brundrett, M.C.; Piché, Y. \& Peterson, R.L. 1984. A new method for observing the morphology of vesicular-arbuscular mycorrhizae. Canadian Journal of Botany 62: 2128-2134.

Calvente, R.; Cano, C.; Ferrol, N.; Azcón-Aguilar, C. \& Barea, J.M. 2004. Analysing natural diversity of arbuscular mycorrhizal fungi in olive tree (Olea europaea L.) plantations and assessment of the effectiveness of native fungal isolates as inoculant for commercial cultivar of olive plantlets. Applied Soil Ecology 26: 11-19.

Caravaca, F.; Alguacil, M.M.; Azcón, R.; Díaz, G. \& Roldán, A. 2004. Comparing the effectiveness of mycorrhizal inoculation and amendment with sugar beet, rock phosphate and Aspergillus niger to enhance field performance of the leguminous shrub Dorycnium pentaphyllum L. Applied Soil Ecology 25: 169-180.

Caravaca, F.; Alguacil, M.M.; Barea, J.M. \& Roldán, A. 2005. Survival of inocula and native AM fungi species associated with shrubs in a degraded mediterranean ecosystem. Soil Biology and Biochemistry 37: 227-233.
Caravaca, F.; Figueroa, D.; Azcón-Aguilar, C.; Barea, J.M. \& Roldán, A. 2003. Medium-terms effects of mycorrhizal inoculation and composted municipal waste addition on the establishment of two Mediterranean shrub species under semiarid field conditions. Agriculture, Ecosystems and Environment 97: 95-105.

Caravaca, F.; Hernández, F.; Gracia, C. \& Roldán, A. 2002. Improvement of rhizosphere aggregate stability of afforested semiarid plants species subjected to mycorrhizal inoculation and compost addition. Geoderma 108: 133-144.

Carrasco, L.; Caravaca, F.; Alvarez-Rogel, J. \& Róldan, A. 2006. Microbial processes in the rhizosphere soil of a heavy metalscontamined Mediterranean salt marsh: a faciliting role of AM fungi. Chemosphere: 104-111.

Chu, E.Y.; Möller, M.R.F. \& Carvalho, J.G. 2001. Efeitos da inoculação micorrízica em mudas de gravioleira em solo fumigado ou não fumigado. Pesquisa Agropecuária Brasileira 36: 671-680.

Costa, C.M.C.; Cavalcante, U.M.T.; Goto, B.T.; Santos, V.F. \& Maia, L.C. 2005. Fungos micorrízicos arbusculares e adubação fosfatada em mudas de mangabeira. Pesquisa Agropecuária Brasileira 40: $225-232$.

Costa, C.M.C.; Maia, L.C.; Cavalcante, U.M.T. \& Nogueira, R.J.M.C. 2001. Influência de fungos micorrízicos arbusculares sobre o crescimento de dois genótipos de aceroleira (Malpighia emarginata D.C.). Pesquisa Agropecuária Brasileira 36: 893-901.

Fidebilus, M.W.; Warting, C.A. \& Stutz, J.C. 2001. Geographic isolates of Glomus increase root growth and whole plant transpiration of Citrus seedlings grown in high phosphorus. Mycorrhiza 10: 231-236.

García-Gil, J.C.; Plaza, C.; Brunetti, G. \& Polo, A. 2004. Effects of sewage sludge amendment on humic acids and microbiological properties of a semiarid Mediterranean soil. Biology and Fertility of Soils 39: 320-328.

Gaur, A. \& Adholeya, A. 2000. Response of three vegetable crops to VAM fungal inoculation in different soils amended with organic matter. Symbiosis 29: 19-31.

Gerdemann, J. W. \& Nicolson, T. H. 1963. Spores of mycorrhizal Endogone species extracted from soil by wet sieving and decanting. Transactions of the British Mycological Society 46: 235-244.

Grisi, B.M. 1978. Método químico de medição da respiração edáfica: alguns aspectos técnicos. Ciência e Cultura 30: 82-88.

Gryndler, M.; Vosátka, M.; Hrselová, H.; Chvátalová, I. \& Jansa, J. 2002. Interaction between arbuscular mycorrhizal fungi and cellulose in growth substrate. Applied Soil Ecology 19: 279-288.

Jenkins, W.R. 1964. A rapid centrifugal-flotation technique for separating nematodes from soil. Plant Disease Report 48: 692.

Lovelock, C.E.; Wright, S.F. \& Nichols, K.A. 2004. Using glomalin as an indicator for arbuscular mycorrhizal hyphal growth: an example from a tropical rain forest soil. Soil Biology and Biochemistry 36: 1009-1012.

Marschner, P.; Kandeler, E. \& Marschner, B. 2003. Structure and function of the soil microbial community in a long-term fertilizer experiment. Soil Biology and Biochemistry 35: 453-461.

Mc Gonigle, T.P.; Miller, M.H.; Evans, D.G.; Fairchild, G.L. \& Swan, J.A. 1990. A new method which gives an objective measure of colonization of roots by vesicular-arbuscular mycorrhizal fungi. New Phytologist 115: 495-501.

Medina, A.; Vassilev, N.; Alguacil, M.M.; Roldán, A. \& Azcón, R. 2004. Increased plant growth, nutrient uptake, and soil enzymatic activities in a desertified Mediterranean soil amended with treated residues and inoculated with native mycorrhizal fungi and a plant growth-promoting yeast. Soil Science 169: 260-270.

Palenzuela, J.; Azcón-Aguilar, C.; Figueroa, D.; Caravaca, F.; Roldán, A. \& Barea, J.M. 2002. Effects of mycorrhizal inoculation of shrubs from Mediterranean ecosystems and composted residue application on transplant performance and mycorrhizal developments in a desertified soil. Biology and Fertility of Soils 36: 170-175. 
Pascual, J.A.; Hernandez, T.; Garcia, C. \& Ayuso, M. 1998. Enzymatic activities in an arid soil amended with urban organic wastes: laboratory experiment. Bioresource Technology 64: 131-138.

Ramos, V.H.V.; Pinto, A.C.Q. \& Rodrigues, A.A. 2001. Introdução e importância socioeconômica. In: M.A.S. Oliveira (ed.). Graviola Produção - Aspectos técnicos. Brasília, EMBRAPA.

Rillig, M.C. 2004. Arbuscular mycorrhizae, glomalin, and soil aggregation. Canadian Journal of Soil Science 36: 355-399.

Roldán, A.; Caravaca, F.; Hernández, M.T.; Garcia, C.; Sánchez-Brito, C.; Velásquez, M. \& Tiscareño, M. 2003. No tillage, crop rotations, and legume corn cropping effects on soil quality characteristics under maize in Patzcuaro watershed (Mexico). Soil and Tillage Research 72: 65-73.

Sieverding, E. 1991. Vesicular-arbuscular Mycorrhiza Management in Tropical Agrosystems. Eschborn, Deutsche Gesellschaft für Technisch Zuzammenarbeit.

Souza, C.A.S.; Corrêa, F.L.O.; Mendonça, V.L. \& Carvalho, J.G. 2003 Crescimento de mudas de gravioleira (Annona muricata L.) em substrato com superfosfato simples e vermicomposto. Revista Brasileira de Fruticultura 25: 453-456.
Swisher, R. \& Carroll, G.C. 1980. Fluorescein diacetate hydrolisis as an estimator of microbial biomass on coniferous needle surfaces. Microbial Ecology 6: 217-226.

Statsoft. 1997. Statistica for Windows. USA, Tulsa.

Tian, C.Y.; Feng, G.; Li, X.L. \& Zhang, F.S. 2004. Different effects of arbuscular mycorrhizal fungal isolates from saline or non-saline on salinity tolerance of plants. Applied Soil Ecology 26: 143-148.

Wamberg, C.; Christensen, S.; Jakobsen, I.; Müller, A.K. \& Sfrensen, S.J. 2003. The mycorrhizal fungus (Glomus intraradices) affects microbial activity in the rhizosphere of pea plants (Pisum sativum). Soil Biology and Biochemistry 35: 1349-1357.

Wuest, S.B.; Caesar-TonThat, T.C.; Wrigth, S.F. \& Williams, J.D. 2005. Organic matter addition, $\mathrm{N}$, and residue burning effects on infiltration biological and physical properties of an intensively tilled silt-loam soil. Soil and Tillage Research 84: $154-167$

Wright, S.F. \& Upadhyaya, A. 1998. A survey of soils for aggregate stability and glomalin, a glycoprotein produced by hyphae of arbuscular mycorrhizal fungi. Plant and Soil 198: 97-107. 